\title{
Study on the Ignition Performance of TaN Transducer by Heat Treatment
}

\author{
Xiaoming Ren ${ }^{1}{ }^{1}$, Kexin Y ${ }^{1}$, Ming Yin ${ }^{1}$, Jianhua Chen ${ }^{1}$, Lan Liu ${ }^{1}$, Jingxin Zhang ${ }^{1}$, Hongzhi Yao ${ }^{1}$, \\ Ruizhen Xie ${ }^{1}$, Wenxin Kan ${ }^{1}$, Yun Zhang ${ }^{2}$ \\ ${ }^{1}$ Science and Technology on Applied Physical Chemistry Laboratory, Xi'an, Shaanxi, China \\ ${ }^{2}$ School of Mechano-Electronic Engineering, XiDian University, Xi'an, Shaanxi, China \\ *Corresponding Author.
}

\begin{abstract}
In order to obtain good $\mathrm{TaN}$ film transducer and reduce its ignition voltage, the influence of the setting position and cooling method of the heat treatment process on the TaN film transducer were compared and analyzed. By measuring the square resistivity, SEM and XRD, the performance of the film before and after heat treatment were characterized. The ignition voltage of the TaN film transducer was tested, and the results showed that the ignition voltage of the heat-treated TaN film transducer could be reduced a lot. It provided a technical way for the microenergy of MEMS pyrotechnics.
\end{abstract}

Keywords: TaN film, ignition voltage, heat treatment, MEMS pyrotechnics

\section{Introduction}

With the deep research of thin film materials and the development of its preparation technology, as well as the development of micro-electro-mechanical system (MEMS) processing technology, it provided technical support for the development of MEMS pyrotechnics. $\mathrm{NiCr}$ as a kind of commonly used thin film resistors, was more and more unable to meet the requirements of high reliability and low energy development of MEMS pyrotechnics due to its disadvantages such as difficult to achieve high resistivity, low bearing power, poor high temperature stability, poor reliability, easy hydrolysis and so on [1-3]. Tantalum nitride (TaN) thin films had excellent electrical properties, stable chemical and thermal properties, good oxidation and corrosion resistance, etc., and had important applications in aerospace, microelectronics, biomedical, power machinery and other fields [4-5]. As a key component of EEDs, TaN film energy commutator based on MEMS technology had obvious advantages in miniaturization and low energy, and had been paid more and more attention [6].

At present, there had been a few reports on the ignition performance of TaN film energy exchange element at home and abroad Vishay Company in the United States disclosed a TaN film initiation chip [7, 8]. Cai et al. prepared $\mathrm{TaN}$ film by DC magnetron sputtering method, and studied the preparation and ignition performance of TaN film ignition bridge [9]. Xiao-ming Ren et al. presented a process for preparing the energy exchangers of TaN thin films, but their reports did not mention the influence of heat treatment on the ignition performance of the energy exchangers of TaN thin films $[10,11]$. However, heat treatment was a necessary step for the preparation of various devices using TaN thin films, so it was particularly important to study the influence of heat treatment on the electrical and ignition properties of Tal thin films. In order to solve the above problems, this paper prepared TaN thin films on the surface of 7740 glass by reactive magnetron sputtering, and then compared two different heat treatment methods. Finally, the ignition properties were compared and analyzed by experiments.

\section{Experiment}

\subsection{Reagents and instruments}

Reagents: silicon wafer, tantalum nitride target (purity 99. 9\%), titanium target (purity 99. 9\%), copper target (purity 99. 99\%), anhydrous ethanol (analytical pure), acetone (analytical pure), photoresist RN-246, photoresist AR-740, deionized water, argon gas (purity 99. 999\%). 
Instruments: ultrasonic cleaning machine; Oven (maximum temperature $250{ }^{\circ} \mathrm{C}$.); KS60VR magnetron sputtering equipment of KENOSISTEC, Italy; VEGA TSS136XM scanning electron microscope (TESCAN, Czech Republic); PRO4 four-probe resistivity measuring instrument instrument from LUCAS.

\subsection{Overall structure design and preparation process of tan energy commutator}

The overall structure of TaN commutator was composed of matrix, intermediate layer, ignition layer and welding pad layer, as shown in Fig. 1. The function of the matrix was as a support carrier, and the matrix material was 7740 glass. The role of the interlayer was to increase the adhesion between the materials, the interlayer material selection titanium; The ignition layer material was TaN. The role of the pad layer improves the electrical conductivity, the pad material was copper. The shape of the bridge area was "1" character bar, as shown in Fig. 2. The size of the bridge area was $0.1 \mathrm{~mm} \times 0.1 \mathrm{~mm}$.

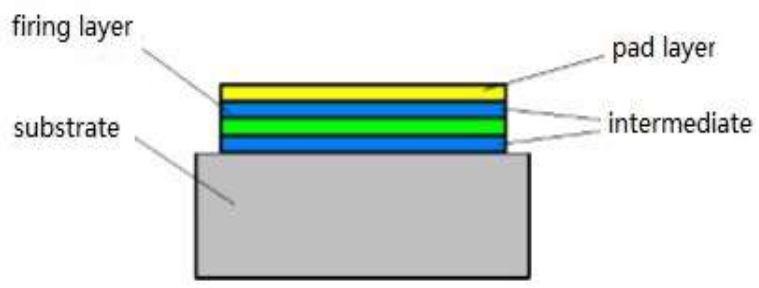

Fig. 1 Structure diagram of TaN transducer

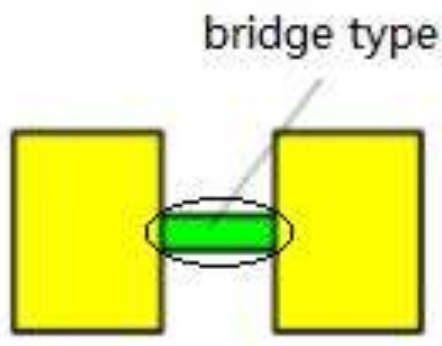

Fig. 2 Diagram of bridge type

The production process of TaN film energy converter was as follows: Firstly, clean 7740 glass, homogenize RN246, bake before, photoetch, bake after, develop, firm film and other processes to produce energy converter graphics, sputter titanium film and TaN film in turn, and prepare TaN film energy converter through stripping process. Then sputtering titanium film and copper film, uniform glue AR-3740, before drying, sleeve engraving, after drying, development, hard film, wet etching and other processes, to produce a welding pad. The production flow chart of TaN commutator was shown in Fig. 3. 


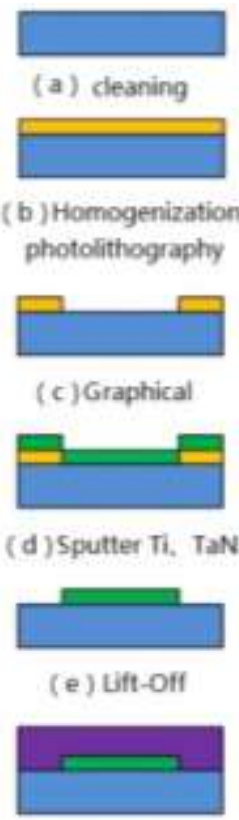

(i) Sputter Ti, Cu

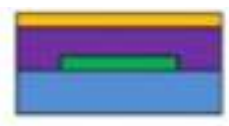

(g) Homogenization photolithography

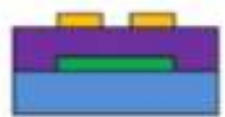

(h) Graphical

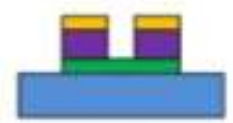

(i) Etching

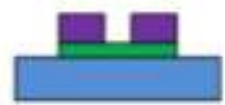

(j) Degumming

Fig. 3. Schematic diagram of production process

\subsection{Characterization methods}

The surface morphology of the film was observed by thermal field emission scanning electron microscopy (SEM). The sheet resistance of the film was measured by four probes. The elements of the film were measured by energy spectrometer. The crystal orientation structure analysis body of the film was measured by XRD.

According to the Langley method used in GJB/ Z377A-94 sensitivity test, the ignition sensitivity of tantalum nitride transducer was tested. $33 \mu \mathrm{F}$ capacitor was used to ignite, and Lead Stephenate was used as the ignition charge. The initiation circuit for the test was shown in Fig. 4.

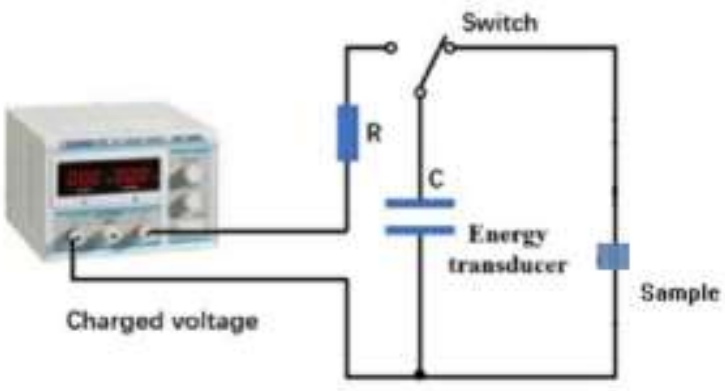

\section{Result and discussion}

Fig. 4. Schematic diagram of initiation circuit

\subsection{Influence of heat treatment on film energy exchangers}

Heat treatment could be placed after the TaN film, or after the preparation of the brazing pad. In the second case, the heat treatment temperature was $250^{\circ} \mathrm{C}$. Fig. 5 shows the comparison of $\mathrm{Cu}$ before and after welding pad. It could be seen from Fig. $5 \mathrm{~b}$ that the $\mathrm{Cu}$ surface had been oxidized and $\mathrm{CuO}$ was produced. It affected the conductivity of the TaN film energy commutator. The heat treatment should be carried out after the preparation of the TaN film and before the preparation of the pad layer. 


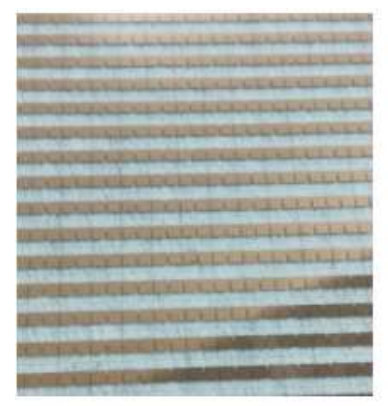

(a) before heat treatment

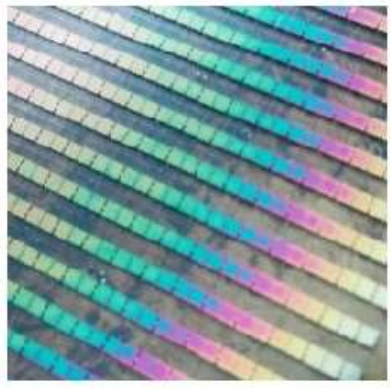

(b)after heat treatment

Fig. 5 Comparison of $\mathrm{Cu}$ pads before and after heat treatment

Cooling methods could be divided into rapid cooling and natural cooling. In this paper, they were tested. The temperature of TaN film drops from $250^{\circ} \mathrm{C}$ to room temperature for 30s. Fig. 6 showed the picture of TaN film after rapid cooling. As could be seen from Fig. 6, TaN film had warped skin phenomenon and even falls off. TaN film decreased from $250^{\circ} \mathrm{C}$ to room temperature for $12 \mathrm{~h}$, but the above situation did not appear. In order to ensure the yield of TaN film energy exchangers, natural cooling should be selected.

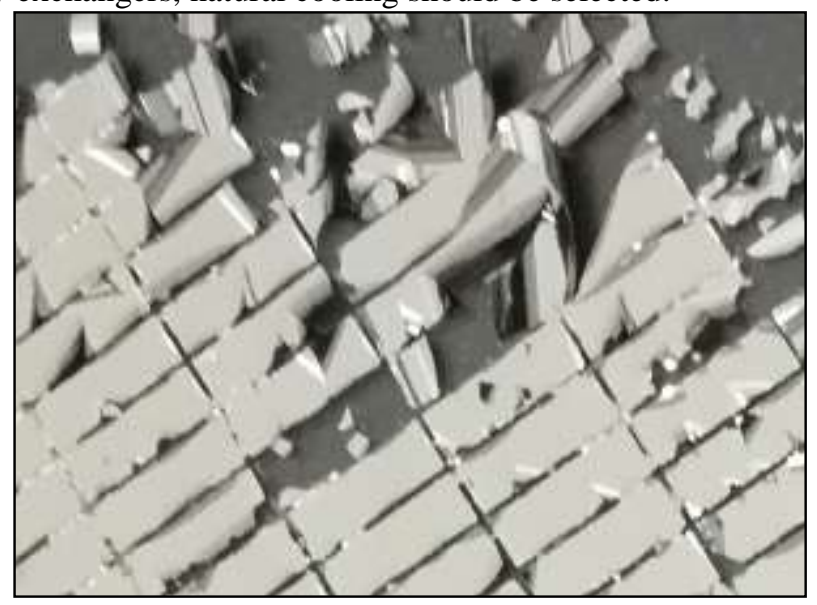

Fig. 6 Picture of tantalum nitride film after rapid cooling

\subsection{The effect of heat treatment on thin film transducer}

The sheet resistance test was performed on the TaN film before and after the heat treatment, and the test results were shown in Table 1. The test results shown that the sheet resistance of the TaN film after heat treatment was slightly reduced.

Table 1 Sheet resistance of the film

\begin{tabular}{|c|c|c|}
\hline Number & $/$ & $\begin{array}{c}\text { Average sheet } \\
\text { resistance } / \Omega / \mathrm{sq}\end{array}$ \\
\hline 1 & $\begin{array}{c}\text { Before heat } \\
\text { treatment }\end{array}$ & 2.388 \\
\hline 2 & $\begin{array}{c}\text { After heat } \\
\text { treatment }\end{array}$ & 2.357 \\
\hline
\end{tabular}

SEM was performed on the TaN films at room temperature and those at $250^{\circ} \mathrm{C}$. See Fig. 7 showed the surface morphology at room temperature $\left(25^{\circ} \mathrm{C}\right)$ after TaN sputtering. It could be seen that the surface of the film layer was uniform continuous, flat and compact, without obvious particles. The surface morphology of the $\mathrm{TaN}\left(250^{\circ} \mathrm{C}\right)$ film was similar to that of the room temperature. It indicated that in this temperature range, the change of TaN film was small. 


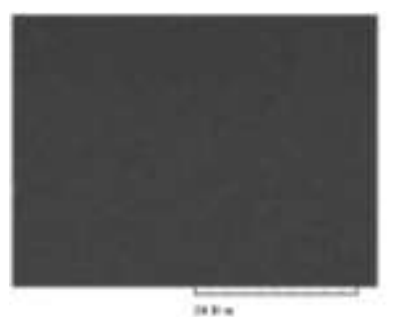

(a) $25^{\circ} \mathrm{C}$

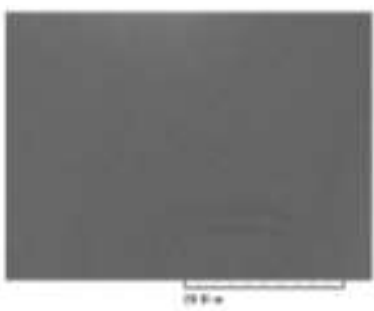

(b) $250^{\circ} \mathrm{C}$

Fig. 7 The SEM of TaN film at different temperatures

The TaN film was analyzed by energy spectrum. The analysis diagram was shown in Fig.8. It shown that the film contained only nitrogen and tantalum elements; The XRD test was performed on the TaN film before $\left(25^{\circ} \mathrm{C}\right)$ and after the heat treatment $\left(250^{\circ} \mathrm{C}\right)$. The XRD diagram was shown in Fig. 9. It could be seen from the figure that the crystal structure of the TaN film before and after the heat treatment had not changed.

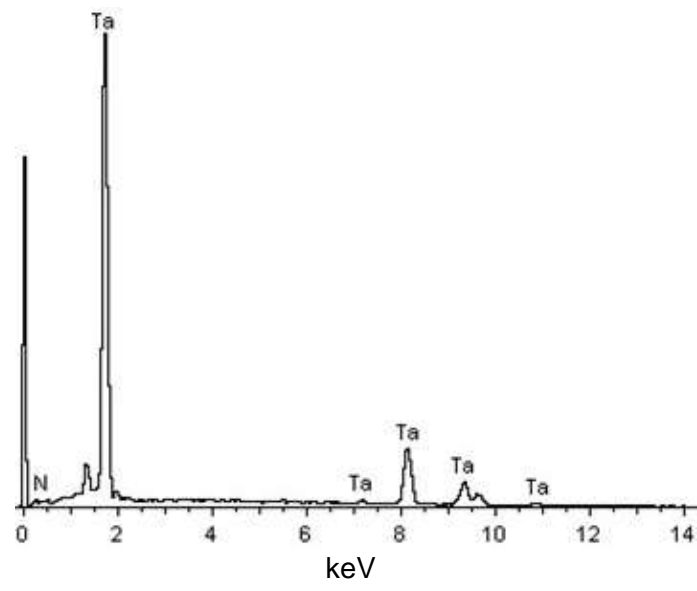

Fig. 8 The analysis diagram of TaN film

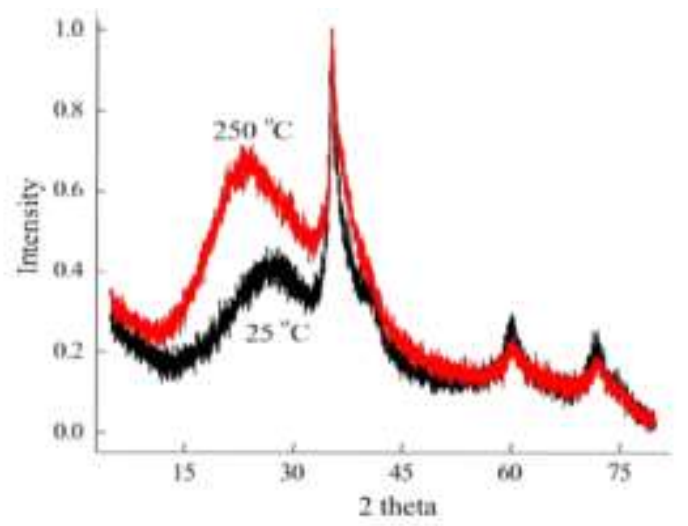

Fig. 9 The XRD diagram of TaN film

3.3 Influence of heat treatment on ignition voltage of tan film commutator

The working principle of TaN converter at low pressure was that the electric energy was converted into heat energy. The external power supply provided electric energy to TaN converter through the solder pad, and the TaN converter converts the electric energy into heat energy, thus initiating the next stage ignition charge. The physical photo of TaN film energy commutator was shown in Fig. 10. The ignition voltage test of TaN film energy commutator after heat treatment and unheated treatment was carried out. The results were shown in Table 2.

It could be seen from Table 2 that heat treatment significantly affected the ignition performance of the TaN film energy commutator, and the ignition voltage of the heat-treated TaN film energy commutator was significantly reduced. Analysis reasons: without overheating treatment of TaN thin film commutator, due to the vacuum 
sputtering the film there were different degrees of defects, including grain accumulation of misfit dislocation, film holes, intrusion, the reaction gas inclusions, etc., these defects on the resistance of the film performance, thermal capacity and the internal stress of membrane layer, adhesion strength, such as a direct impact. Therefore, in the process of heating and conduction of the TaN thin film commutator without overheating treatment, the current would cause the composition change or a small amount of grain rearrangement in the film, resulting in a larger ignition voltage.

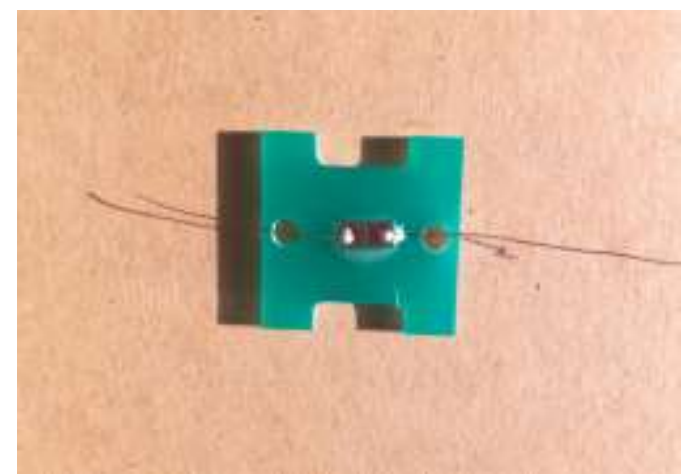

Fig.10 The physical photo of TaN film energy commutator

Table 2 Ignition sensitivity test results for transducer

\begin{tabular}{|c|c|c|}
\hline Number & 1 & 2 \\
\hline Temperature $/{ }^{\circ} \mathrm{C}$ & 25 & 250 \\
\hline Quantity & 15 & 15 \\
\hline Ignitionvoltage/V & 10.5 & 6.4 \\
\hline Standarddeviation/V & 0.52 & 0.18 \\
\hline $99.9 \%$ ignitionvoltage/V & 12.1 & 6.9 \\
\hline $0.1 \%$ ignitionvoltage/V & 8.9 & 5.8 \\
\hline
\end{tabular}

\section{Conclusion}

The heat treatment process should be carried out after the preparation of the TaN film energy commutator and before the preparation of the pads. Reduce the temperature from 250 to room temperature using natural cooling, can provide yield; After heat treatment, the surface morphology of the film had not changed, the crystal structure of the film had not changed, the sheet resistance of the TaN film was slightly reduced. Heat treatment significantly affects the ignition performance of TaN film energy commutator. After heat treatment, the ignition voltage of TaN film energy commutator was significantly reduced, which provides a technical way for the low energy of TaN film energy commutator.

\section{References}

[1] N.M. Phuong, D.J. Kim, B.D. Kang, et al., "Effect of chromium concentration on the electrical properties of nicr thin films resistor deposited at room temperature by magnetron cosputtering technique," Journal of the Electrochemical Society, vol. 153, pp. 27, 2006.

[2] L. Lai, J. Wang, H. Wang, et al. "Structures and Properties of C-D oped NiCr Thin Film Deposited by Closed-Field Unbalanced Magnetr on Sputtering," Journal of Electronic Materials, vol. 46, pp. 11, 2017 (in Chinese).

[3] J.F. Fang, M.J. Ding, Y.L. Feng, et al. "Effect of annealing temperature on the performance of tantalum nitride film resistor," Guangzhou Chemical Industry, vol. 46, pp. 39. 2018.

[4] H.C. Jiang, C.J. Wang, W.L. Zhang, et al. "Composition control and its electric properties of TaNx thin films,” Mod. Phys. Lett. B, vol. 24, no. 905, 2010.

[5] Z.Y. Jia, J.Q. Zhu, S.C. Cao. "Study on Preparation of Tantalum Nitride Diffusion Barrier by Reactive Magnetron Sputtering," Chinese Journal of Vacuum Science and Technology, vol. 32, pp.751, 2012.

[6] R.Z. Xie, X.M. Ren, K.X. Wang, et al., "Preparation of NiCr Bridge Film Transducer," Chinese Journal of Energetic Materials, vol. 19, pp. 584, 2011. 
[7] Khan, A. Ibrarb, W. Ahmed, et al. "Synthetic approaches,functionalization and therapeutic potential of quinazoline and quinazolinone skeletons: the advances continue,” Eur. J. Med. Chem, vol. 90, pp. 124, 2015.

[8] F.F. Liu, Y. Tang, W.L. Zhang, H.C. Jiang. "The effect of heat treatment on the electrical properties of tan films," Electronic Components and Materials, vol. 30, pp. 47, 2011.

[9] X.Y. Cai, H.C. Jiang, Y.C Yan, Y.X. Zhang, et al., "(B/Ti)n/TaN film ignition bridge preparation and ignition performance research,” Chinese Journal of Energetic Materials, vol. 23, pp. 265, 2015.

[10] X.M. Ren, Q. Su, R.Z. Xie, Y. Xue, et al., "Study on the Preparation Technology of Tantalum Nitride Transducer,” Initiators \& Pyrotechnics, vol. 1, pp. 26, 2020.

[11] X.M. Ren, L. Liu, K.X. Yu, et al., "Research on Low Energy of Tantalum Nitride Transducer," Initiators \& Pyrotechnics, vol. 1, pp. 5, 2021. 